\title{
Is Prenatal Diagnosis Necessary for Fetal Isolated Nasal Bone Absence or Hypoplasia?
}

\author{
Feng Zhang $\mathbb{D}^{\prime}$ \\ Wei Long $\mathbb{D}^{\prime}$ \\ Qin Zhou' \\ Jing Wang' \\ Ye Shi ${ }^{1}$ \\ Jianbing Liu' ${ }^{1} *$ \\ Qiuwei Wang ${ }^{2, *}$ \\ 'Department of Medical Genetics, \\ Changzhou Maternity and Child Health \\ Care Hospital Affiliated to Nanjing \\ Medical University, Changzhou, Jiangsu \\ Province, People's Republic of China; \\ ${ }^{2}$ Clinical Laboratory, Changzhou \\ Childrens Hospital Affiliated to Nantong \\ Medical University, Changzhou, Jiangsu \\ Province, People's Republic of China \\ *These authors contributed equally to \\ this work
}

Correspondence: Jianbing Liu Department of Medical Genetics, Changzhou Maternity and Child Health Care Hospital Affiliated to Nanjing Medical University, Changzhou, Jiangsu Province, People's Republic of China Tel +86 05।9-8858I983

Email liujb2222@I26.com

Qiuwei Wang

Clinical Laboratory, Changzhou

Children's Hospital Affiliated to Nantong

Medical University, Changzhou, Jiangsu

Province, People's Republic of China

Tel +86 0519-69808280

Email qiuwei_wang@ntu.edu.cn
Purpose: This study aimed to explore the value of chromosomal microarray analysis (CMA) and whole exome sequencing (WES) in the prenatal diagnosis of fetal isolated nasal bone absence (INBA) or isolated nasal bone hypoplasia (INBH). We hope to provide additional relevant information for clinical counseling.

Patients and Methods: From November 1, 2018, to March 1, 2020, 55 pregnant women with isolated nasal bone dysplasia were admitted to the Changzhou Maternity and Child Health Care Hospital. Based on the degree of abnormality, the patients were divided into two groups: INBA and INBH. CMA was performed on all patients. The clinical data and prenatal genetic diagnoses of the two groups were retrospectively analyzed. According to the requirements of WES for samples, 12 cases with negative CMA results were selected for the WES test.

Results: A total of 55 cases with INBA or INBH met the inclusion criteria. In 35INBA fetuses, there was one case of trisomy 21 and one case of $10 \mathrm{q} 11.22$ deletion $(5.7 \mathrm{Mb})$, and the abnormality rate was $5.71 \%(2 / 35)$. Compared with INBA fetuses, the abnormality rate was increased in the fetuses with INBH $[15.00 \%$ (3/20)] (15.00\% vs 5.71\%); there was one case of 1q21.1 duplication (1.3Mb), one case of Xp22.31 duplication (1.67Mb), and one case of $4 \mathrm{p}$ deletion $(7.6 \mathrm{Mb})$. In a later retrospective study, two pathogenic variants were identified in two cases after the WES test; the abnormality rate was $16.67 \%(2 / 12)$, which involved RUNX2 and $\mathrm{CDH} 4$ genes, respectively.

Conclusion: A preliminary study confirmed that molecular prenatal diagnosis should be performed in fetuses with INBA or INBH. CMA followed by WES is an effective method. Keywords: chromosomal microarray, whole exome sequencing, isolated nasal bone absence, isolated nasal bone hypoplasia

\section{Introduction}

Nasal bone abnormalities, including nasal bone absence and hypoplasia, have become the focus of many studies. Previous studies showed that the incidence of fetal nasal bone absence or hypoplasia was $0.5-4.5 \%$ among fetuses with a normal karyotype. ${ }^{1-3}$ Upon prenatal ultrasound examination, it is noted that nasal bone abnormalities are often accompanied by other structural abnormalities or soft markers, such as endocardial cushion defect, increased nuchal fold thickness ( $\geq 6 \mathrm{~mm}$ ), short femur or humerus, ventriculomegaly ( $\geq 10 \mathrm{~mm}$ ), intestinal hyperechogenicity, and echogenic intracardiac focus among others. ${ }^{1}$ It is widely known that nasal bone absence or hypoplasia is associated with fetal chromosomal abnormalities, such as trisomy $13,18,21$, and Turner syndrome. In particular, it is closely related to trisomy 21 , with a likelihood ratio of 11.6 to $50.5 \cdot{ }^{1-5}$ Rare 
conditions have also been reported to be associated with nasal bone absence or hypoplasia, such as Cri du chat (5p-) syndrome, Wolf-Hirschhorn syndrome (4p-), and Fryns Syndrome. ${ }^{6-8}$ Ultrasound measurement of nasal bone length has become a routine part of prenatal care during the first or second trimester, and is recommended for all pregnant women. However, it is difficult to measure fetal nasal bone length during early pregnancy because of unclear imaging, but image clarity improves as the fetus grows. In the second trimester, the nasal bone is clearly visible and can be more easily measured and evaluated.

In the recent years, Europe, the United States, and China have issued statements recommending chromosome microarray analysis (CMA) as the preferred diagnostic method for fetal genetic detection of prenatal ultrasound abnormalities. $^{9-11}$ In clinical practice, pregnant women with nasal bone absence or hypoplasia in association with other structural abnormalities are generally recommended to undergo prenatal karyotype analysis or CMA tests after clinical counseling.

However, even when the karyotype or CMA is normal, fetal genetic abnormalities may still occur. ${ }^{12,13}$ It is necessary to develop new efficient screening technologies to improve the detection rate of chromosomal abnormalities. Whole exome sequencing (WES) is used not only to detect known disease-related genes, but also to discover new disease-related genes, and diagnoses nearly $20 \%$ of rare diseases. ${ }^{14} \mathrm{~A}$ meta-analysis by Clark et al reported that the diagnostic and clinical utility of WGS/WES was greater than that of CMA in children with suspected genetic diseases. ${ }^{12}$ During prenatal diagnosis, even when karyotype or CMA is normal, fetal genetic abnormalities may still occur, while WES may improve the identification of genetic disorders in fetuses with structural abnormalities. ${ }^{15,16}$ Currently, studies have focused on fetal ultrasound structural abnormalities and/or soft marker abnormalities. Few studies have examined the prenatal diagnosis of single soft marker abnormalities using CMA or WES. Whether prenatal diagnosis of fetal isolated nasal bone absence or hypoplasia is necessary and which technology should be used for diagnosis remains unclear. This makes clinical consultations complicated.

However, whether pregnant women with isolated nasal bone absence (INBA) or isolated nasal bone hypoplasia (INBH) should be advised to undergo prenatal diagnosis remains to be determined. The extent of the risk of genetic variation in these fetuses remains unclear. The aim of this study was to review the prenatal diagnosis and outcomes of pregnancies with INBA or INBH in the second trimester, in order to explore the value of CMA and WES in the diagnosis of INBA or INBH. We hope to provide additional relevant information for clinical counseling.

\section{Patients and Methods}

\section{Patients and Design}

The study design and protocol were reviewed and approved by the ethics committee of the Changzhou Maternity and Child Health Care Hospital affiliated with Nanjing Medical University (No. 2017003). All participants received genetic counseling and provided informed consent prior to testing according to the guidelines of the Declaration of Helsinki.

From November 2018 to March 2020, 55 pregnant women were reported to have fetal nasal bone absence/ hypoplasia after ultrasound examination in the second trimester, including 35 cases of INBA and 20 cases of INBH. Patients were 25-37 years old, and 23 weeks (and 5 days) to 29 weeks (and 2 days) pregnant, respectively. The characteristics of all women are shown in Table 1. They were recalled for genetic counseling, and prenatal diagnosis was accepted. All patients underwent traditional karyotype analysis and the CMA test. Moreover, with the development and wide application of WES, 12 cases with negative CMA results agreed to undergo further testing using WES.

\section{Nasal Bone Measurement}

Routine ultrasound examination of the abdomen was performed to show the median sagittal section of the fetal head, so that the fetal head was facing the probe. The nasal bone was measured at the level of synostosis using the method and reference range as previously described by Sonek et al. ${ }^{17}$ The nasal bone was considered absent if it was not visualized on any appropriate view. Cases with sagittal sections of the fetuses showed that one or both

Table I The Characteristics of INBA and INBH

\begin{tabular}{|l|l|l|}
\hline & INBA (n=35) & INBH (n=20) \\
\hline Maternal age(y) & $28.06 \pm 4.39$ & $27.10 \pm 4.50$ \\
Gestational age at ultrasound(w) & $23.11 \pm 3.62$ & $23.14 \pm 3.83$ \\
Gestational age at CMA(w) & $24.58 \pm 1.99$ & $24.30 \pm 2.18$ \\
Singleton & 35 & 20 \\
High risk of prenatal screening & $\mathrm{I}$ & 0 \\
Low risk of prenatal screening & 32 & 19 \\
No screening & 2 & 1 \\
\hline
\end{tabular}

Abbreviations: INBA, isolated nasal bone absence; INBH, isolated nasal bone hypoplasia. 
sides of the nasal bone length below the 2.5 th percentile were considered hypoplastic. ${ }^{18}$

\section{Chromosomal Microarray Analysis for Prenatal Diagnosis}

Amniotic fluid $(10 \mathrm{~mL})$ was collected with informed consent, and genomic DNA was extracted using a QIAamp DNA Mini Kit (Qiagen, Valencia, CA, USA). Using an Affymetrix CytoScan 750K Array chip, DNA (250 ng) was amplified, labeled, and hybridized according to the manufacturer's protocol. The data were analyzed using the Chromosome Analysis Suite v3.2 (ChAs) software package, and the pathogenicity was analyzed according to OMIM, UCSC, DECIPHER, ISCA, and DGV databases.

\section{Whole Exome Sequencing for Prenatal Diagnosis}

After the genomic DNA was processed by the M220 breaker (American Covaris), the SureSelect hereditary disease gene detection (2742 genes) kit (American Agilent) was used to build the library, and the HiSeq 2500 System (American Illumina company) was used for highthroughput sequencing. The data were read by NextGENe software and uploaded to Ingenuity Variant Analysis TM software for bioinformatic analysis.

\section{Statistical Analyses}

The parameters of age and gestational age were expressed as median (M), 2.5th percentile (P2.5), and 97.5th percentile (P97.5). The chi-square test or Fisher's exact test was used to compare differences between the two groups. All significance tests were two-sided and a p-value $<0.05$ was considered statistically significant.

\section{Results}

In this study, 55 pregnant women with INBA or INBH underwent CMA prenatal diagnosis. WES was performed in 12 cases with negative CMA results. The characteristics of INBA and INBH are listed in Table 1.

After the prenatal CMA test, five women with fetal INBA or INBH were confirmed to have chromosomal abnormalities; the abnormality rate was $9.10 \%(5 / 55)$ (Table 2). Of the 55 participants, $15.00 \%(3 / 20)$ had INBH and 5.71\% (2/35) had INBA. Although the abnormality rate of INBH was slightly higher, there was no significant difference between the two groups. One case of trisomy 21 with INBA was found, and the fetus had no
Table 2 The Results of CMA Prenatal Diagnosis of INBA and INBH

\begin{tabular}{|l|l|l|l|}
\hline & $\begin{array}{l}\text { INBA } \\
\text { (n =35) }\end{array}$ & $\begin{array}{l}\text { INBH } \\
\text { (n =20) }\end{array}$ & p \\
\hline Aneuploidies, n (\%) & I (2.86\%) & $0(0 \%)$ & \\
Micro-duplication/deletion, n (\%) & I (2.86\%) & $3(15.00 \%)$ & \\
Total abnormalities, n (\%) & $2(5.71 \%)$ & $3(15.00 \%)$ & 0.10 \\
\hline
\end{tabular}

Abbreviations: INBA, isolated nasal bone absence; INBH, isolated nasal bone hypoplasia.

other ultrasound abnormalities. The mother's prenatal screening results did not suggest any abnormalities. Four cases were reported as micro-duplication/deletion, involving chromosomes $1,4,10$, and $\mathrm{X}$, and the fragment sizes ranged from $1.1 \mathrm{Mb}$ to $7.6 \mathrm{Mb}$. According to the literature and database, ${ }^{14,19-25}$ they were identified as three cases with pathogenic copy number variants (CNVs) and one with unclear clinical significance. After genetic counseling, the mothers chose to undergo clinical intervention combined with the clinical phenotype and penetrance of CNV, as shown in Table 3. Notably, case 3 was confirmed as $\operatorname{arr}[\mathrm{hg} 19] 1 \mathrm{q} 21.1 \mathrm{q} 21.2(146,586,249-147,844,778)$ $\mathrm{x} 3$, which showed $1.3 \mathrm{Mb}$ duplication in the 1q21.1q21.2 region, containing 10 OMIM genes. Many studies have reported that 1q21.1 duplication syndrome complicates clinical manifestations and leads to incomplete penetrance. ${ }^{20,21}$ The mother decided to continue the pregnancy and successfully delivered a boy. After 6 months, the infant showed no signs of intellectual disability or other manifestations.

Twelve patients underwent a prenatal WES test after obtaining negative results of CMA. As a result, two cases were confirmed as pathogenic $\mathrm{CNVs}$, and the additional abnormal rate of WES was $16.67 \%(2 / 12)$ (Table 4).

Case 5 was detected as INBA by ultrasound at 24 weeks gestation. After genetic counseling, the mother agreed to undergo the CMA test after amniocentesis, and the result was negative. The mother then underwent the WES test, and the result showed a splice-site mutation (c. $1021+1 \mathrm{G}>\mathrm{T}$ ) involving the RUNX2 gene, which was reported to cause cleidocranial dysplasia (CCD; MIM119600). CCD is a congenital autosomal dominant genetic disease with high penetrance and variable expressivity. ${ }^{26}$ The most common clinical manifestations are delayed closure of the fontanel, widening of the cranial suture, dysplasia of the clavicle and teeth, and short stature. $^{27}$ The runt-related transcription factor 2 (RUNX2; MIM 600211) gene is known as the main 
Table 34 Cases with Micro-Duplication/Deletion After CMA Test

\begin{tabular}{|c|c|c|c|c|c|c|c|c|c|}
\hline \multirow[t]{2}{*}{ Case } & \multirow{2}{*}{$\begin{array}{l}\text { MA } \\
(y)\end{array}$} & \multirow{2}{*}{$\begin{array}{l}\text { GA } \\
(w)\end{array}$} & \multirow{2}{*}{$\begin{array}{l}\text { Ultrasonic } \\
\text { Diagnosis }\end{array}$} & \multirow{2}{*}{$\begin{array}{l}\text { Chromosome } \\
\text { Region }\end{array}$} & \multicolumn{4}{|c|}{ CMA Test } & \multirow{2}{*}{$\begin{array}{l}\text { Pregnancy } \\
\text { Outcome }\end{array}$} \\
\hline & & & & & $\begin{array}{l}\text { Variation } \\
\text { Section }\end{array}$ & $\begin{array}{l}\text { Fragment } \\
\text { Size }\end{array}$ & $\begin{array}{l}\text { Variant } \\
\text { Type }\end{array}$ & Classification & \\
\hline 1 & 30 & 24.5 & INBA & $10 q 11.22$ & $\begin{array}{l}46,225,349- \\
51,904,377\end{array}$ & $5.7 \mathrm{Mb}$ & Deletion & Pathogenic & ToP \\
\hline 2 & 25 & 26.4 & INBH & $|q 2| .|q 2| .2$ & $\begin{array}{l}\mid 46,586,249- \\
\mid 47,844,778\end{array}$ & $1.3 \mathrm{Mb}$ & Duplication & Pathogenic & $\begin{array}{l}\text { LB, } 2750 \mathrm{~g}, 37+5 \\
\text { weeks GA } \\
\text { Normal newborn } \\
\text { examination }\end{array}$ \\
\hline 3 & 27 & 25.5 & INBH & $4 p 16.3 p 16.1$ & $\begin{array}{l}1,124,732- \\
8,721,580\end{array}$ & $7.6 \mathrm{Mb}$ & Deletion & Pathogenic & ToP \\
\hline 4 & 27 & 25 & $\mathrm{INBH}$ & Xp22.31 & $\begin{array}{l}6,455,151- \\
8,127,579\end{array}$ & $1.1 \mathrm{Mb}$ & Duplication & VUS & $\begin{array}{l}\text { LB, } 3 \mid 40 \mathrm{~g}, 40 \\
+5 \text { weeks GA } \\
\text { Normal newborn } \\
\text { examination }\end{array}$ \\
\hline
\end{tabular}

Abbreviations: MA, maternal age; GA, gestational age; INBA, isolated nasal bone absence; INBH, isolated nasal bone hypoplasia; CMA, chromosomal microarray; VUS, variants of uncertain significance; ToP, termination of pregnancy; LB, live birth.

Table 42 Cases with Monogenic Diseases After WES

\begin{tabular}{|l|l|l|l|l|l|}
\hline Case & MA(y) & Ultrasonic Diagnosis & WES Results & Inheritance & Disease Association \\
\hline 5 & 29 & INBA & RUNX2 c.102I+IG>T chr6-45480I45 & AD & Cleidocranial Dysplasia \\
6 & 31 & INBA & CDH4c.48I9het_delG, p.VI607Sfs & AD De novo & Sifrim-Hitz-Weiss syndrome \\
\hline
\end{tabular}

Abbreviations: MA, maternal age; INBA, isolated nasal bone absence; AD, autosomal dominant inheritance.

pathogenic gene of CCD. It was found that this mutation had not been previously reported. The mother gave birth to a boy, who was followed up for half a year, and showed no signs of intellectual disability or other manifestations.

Case 6 was a fetus with an ultrasonic diagnosis of INBA to our hospital for genetic counseling at 25 weeks 'gestation. WES was performed in the case of a negative CMA result. WES result demonstrated a frameshift mutation (c.581-4_581-1delGCAG), involved the CDH4 gene, which was reported to cause Sifrim-Hitz-Weiss syndrome (SIHIWES; MIM617159). Sifrim-Hitz-Weiss syndrome is an autosomal dominant intellectual developmental disorder with variable congenital defects affecting other systems, including cardiac, skeletal, and urogenital disorders. Patients may have short stature, enlarged head circumference, hearing loss, and nonspecific dysmorphic facial features. $^{28}$ The mutation was identified as a novel frameshift mutation after parental verification (Figure 1). During the follow-up of the pregnancy outcome, it was found that the patient gave birth to a girl at term, and the the infant's nasal bone appeared normal. The infant was followed up for ten months, and showed no signs of intellectual disability or other manifestations.

\section{Discussion}

In addition to its known association with trisomy 21, a hypoplastic nasal bone may be an objective marker of facial dysmorphism associated with clinically relevant CNVs. ${ }^{4-6}$ Compared to traditional G-band karyotyping, CMA is a high-resolution genomic technology that can reveal submicroscopic imbalances or CNVs. ${ }^{16,29}$ Numerous studies have shown that CMA provides additional information in 6-7\% of pregnancies with abnormal ultrasound findings, and as such is now the recommended first-tier genetic test. In the study, four cases $(7.27$ (4/55)) were detected with clinically relevant CNVs, which may go undetected by standard karyotype analysis, and the incidence was consistent with the results reported in previous studies. ${ }^{30,31}$ Two patients chose to terminate their pregnancy voluntarily, while the other two opted to continue the pregnancy after clinical counseling. The later flow-up showed no signs of intellectual disability or other manifestations. 


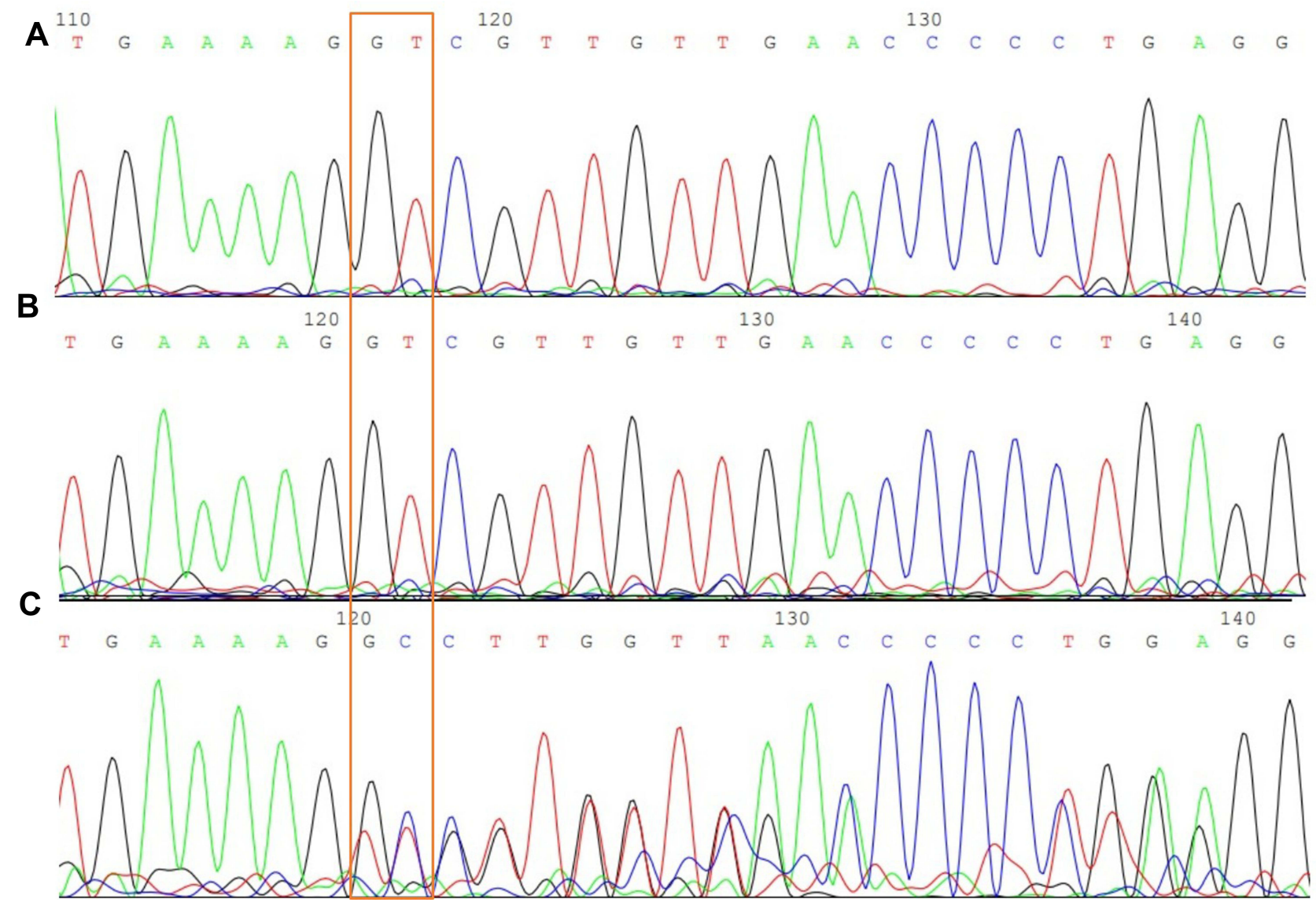

Figure I The result of WES in case b due to parental verification.

Notes: Sequencing results for patient $(\mathbf{C})$, patient's father $(\mathbf{B})$, and mother $(\mathbf{A})$. A heterozygous deletion (c.48I9het_delG) in CDH4 was found in the fetus and was not detected in her parents.

WES can detect and indels in exon regions, as well as CNVs and gene structure variation. It can be used not only to detect known disease-related genes, but also to discover new disease-related genes; it diagnoses nearly $20 \%$ of rare diseases. ${ }^{32}$ During the study, two cases of pathogenic variants were found after WES; the incidence was $16.67 \%(2 / 12)$, and the abnormal detection rate improved after CMA detection.

However, onelimitation of WES in INBA or INBH in this study was that the test was carried out for the purpose of the research. Although the sequencing results suggested that there were pathogenic mutations in two cases, the pregnant women had to continue the pregnancy because the prenatal CMA results were normal. Another limitation was that a number of variants would not be found to lack phenotypes during pregnancy or even after birth. We will continue to follow up these two cases, and they may show clinical phenotypes consistent with the disease-causing genes as they age.
In conclusion, this preliminary study suggested that the abnormality rate was $7.27 \%$ after prenatal CMA diagnosis in fetal isolated nasal bone absence or hypoplasia. An additional abnormality rate of $16.67 \%$ was found in further WES tests. The present results confirm that molecular prenatal diagnosis should be performed in fetal isolated nasal bone absence or hypoplasia, as it may be useful in improving the clinical management of pregnancies and in improving the reproductive decisions of affected families.

\section{Acknowledgments}

We thank all of the participants for their contribution to this study.

\section{Funding}

This work was supported by Changzhou Key Laboratory of High-tech Research (CM20193009), Project funding for the training of high-level health professionals in Changzhou (2016CZLJ013), and key discipline of 
maternal and child health care in Jiangsu Province (FXK201754).

\section{Disclosure}

The authors have no conflicts of interest to disclose.

\section{References}

1. Du Y, Ren Y, Yan Y, Cao L. Absent fetal nasal bone in the second trimester and risk of abnormal karyotype in a prescreened population of Chinese women. Acta ObstetGynecol Scand. 2018;97(2):180-186. doi:10.1111/aogs.13263

2. Agathokleous M, Chaveeva P, Poon LC, Kosinski P, Nicolaides KH. Meta-analysis of second-trimester markers for trisomy 21. Ultrasound Obstet Gynecol. 2013;41(3):247-261. doi:10.1002/ uog. 12364

3. Manegold-Brauer G, Maymon R, Shor S, et al. Down's syndrome screening at 11-14 weeks' gestation using prenasal thickness and nasal bone length. Arch Gynecol Obstet. 2019;299(4):939-945. doi:10.1007/s00404-019-05083-2

4. Moreno-Cid M, Rubio-Lorente A, Rodríguez MJ, et al. Systematic review and meta-analysis of performance of second-trimester nasal bone assessment in detection of fetuses with Down syndrome. Ultrasound Obstet Gynecol. 2014;43(3):247-253. doi:10.1002/ uog. 13228

5. Gu YZ, Nisbet DL, Reidy KL, Palma-Dias R. Hypoplastic nasal bone: a potential marker for facial dysmorphism associated with pathogenic copy number variants on microarray. Prenat Diagn. 2019;39(2):116-123. doi:10.1002/pd.5410

6. Dukhovny S, Wilkins-Haug L, Shipp TD, Benson CB, Kaimal AJ, Reiss R. Absent fetal nasal bone: what does it mean for the euploid fetus? J Ultrasound Med. 2013;32(12):2131-2134. doi:10.7863/ ultra.32.12.2131

7. Sherer DM, Eugene P, Dalloul M, et al. Second-trimester diagnosis of cri du chat $(5 \mathrm{p}-)$ syndrome following sonographic depiction of an absent fetal nasal bone. J Ultrasound Med. 2006;25(3):387-388. doi:10.7863/jum.2006.25.3.387

8. Xing Y, Holder JL Jr, Liu Y, et al. Prenatal diagnosis of Wolf-Hirschhorn syndrome: from ultrasound findings, diagnostic technology to genetic counseling. Arch Gynecol Obstet. 2018;298 (2):289-295. doi:10.1007/s00404-018-4798-1

9. On Genetics, Gynecologists Committee. Committee Opinion No. 581: the use of chromosomal microarray analysis in prenatal diagnosis. ObstetGynecol. 2013;122(6):1374-1377.

10. American College of Obstetricians and Gynecologists and others. ACOG Committee Opinion No. 446: array comparative genomic hybridization in prenatal diagnosis. Obstet Gynecol. 2009;114 (5):1161-1163. doi:10.1097/AOG.0b013e3181c33cad

11. Miller DT, Adam MP, Aradhya S, et al. Consensus statement: chromosomal microarray is a first-tier clinical diagnostic test for individuals with developmental disabilities or congenital anomalies. $\mathrm{Am}$ J Hum Genet. 2010;86(5):749-764. doi:10.1016/j.ajhg.2010.04.006

12. Clark MM, Stark Z, Farnaes L, et al. Meta-analysis of the diagnostic and clinical utility of genome and exome sequencing and chromosomal microarray in children with suspected genetic diseases. NPJ GenomMed. 2018;3:16.

13. Yang $X$, Huang LY, Pan M, et al. Exome sequencing improves genetic diagnosis of fetal increased nuchal translucency. PrenatDiag. 2020;40(11):1426-1431.

14. Stankiewicz P, Kulkarni S, Dharmadhikari AV, et al. Recurrent deletions and reciprocal duplications of 10q11.21q11.23 including CHAT and SLC18A3 are likely mediated by complex low-copy repeats. Hum Mutat. 2012;33(1):165-179. doi:10.1002/humu.21614
15. Petrovski S, Aggarwal V, Giordano JL, et al. Whole-exome sequencing in the evaluation of fetal structural anomalies: a prospective cohort study. Lancet. 2019;393(10173):758-767. doi:10.1016/ S0140-6736(18)32042-7

16. Levy B, Wapner R. Prenatal diagnosis by chromosomal microarray analysis. FertilSteril. 2018;109(2):201-212.

17. Sonek JD, McKenna D, Webb D, Croom C, Nicolaides K. Nasal bone length throughout gestation: normal ranges based on 3537 fetal ultrasound measurements. Ultrasound Obstet Gynecol. 2003;21 (2):152-155. doi:10.1002/uog.41

18. Hung JH, Fu CY, Chen CY, Chao KC, Hung J. Fetal nasal bone length and Down syndrome during the second trimester in a Chinese population. J Obstet Gynaecol Res. 2008;34(4):518-523.

19. Ghai SJ, Shago M, Shroff M, Yoon G. Cockayne syndrome caused by paternally inherited $5 \mathrm{Mb}$ deletion of 10q11.2 and a frameshift mutation of ERCC6. Eur J Med Genet. 2011;54(3):272-276. doi:10.1016/ j.ejmg.2011.02.008

20. Brunetti-Pierri N, Berg JS, Scaglia F, et al. Recurrent reciprocal 1q21.1 deletions and duplications associated with microcephaly or macrocephaly and developmental and behavioral abnormalities. Nat Genet. 2008;40(12):1466-1471. doi:10.1038/ng.279

21. Gourari I, Schubert R, Prasad A. 1q21.1 Duplication syndrome and epilepsy: case report and review. Neurol Genet. 2018;4(1):e219. doi:10.1212/NXG.0000000000000219

22. Guy C, Wang X, Lu X, Lu J, Li S. Clinical report: variable phenotypic expression in a large sibling cohort with a deletion of $4 \mathrm{p} 16.1$. Clin Case Rep. 2016;4(10):913-918. doi:10.1002/ccr3.638

23. Battaglia A, Carey JC, South ST. Wolf-Hirschhorn syndrome: a review and update. Am J MedGenet SeminMedGenet. 2015;169 (3):216-223.

24. Furrow A, Theisen A, Velsher L, et al. Duplication of the STS region in males is a benign copy-number variant. Am J MedGenet SeminMedGenet. 2011;155a(8):1972-1975.

25. Li F, Shen Y, Köhler U, et al. Interstitial microduplication of Xp22.31: causative of intellectual disability or benign copy number variant? Eur JMedGnet. 2010;53(2):93-99.

26. Guo YW, Chiu CY, Liu CL, Jap TS, Lin LY. Novel mutation of RUNX2 gene in a patient with cleidocranial dysplasia. Int JClin ExpPathol. 2015;8(1):1057-1062.

27. Muzio LL, Tetè S, Mastrangelo F, et al. A novel mutation of gene CBFA1/RUNX2 in cleidocranial dysplasia. Ann ClinLabSci. 2007;37 (2):115-120.

28. Weiss K, Terhal PA, Cohen L, et al. De novo mutations in CHD4, an ATP-dependent chromatin remodeler gene, cause an intellectual disability syndrome with distinctive dysmorphisms. Am J HumGenet. 2016;99(4):934-941. doi:10.1016/j.ajhg.2016.08.001

29. Ganapathi M, Nahum O, Levy B. Prenatal diagnosis using chromosomal SNP microarrays. Methods Mol Biol. 2019;1885:187-205.

30. Callaway JL, Shaffer LG, Chitty LS, Rosenfeld JA, Crolla JA. The clinical utility of microarray technologies applied to prenatal cytogenetics in the presence of a normal conventional karyotype: a review of the literature. PrenatDiag. 2013;33(12):1119-1123.

31. Wapner RJ, Martin CL, Levy B, et al. Chromosomal microarray versus karyotyping for prenatal diagnosis. $N$ Engl J Med. 2012;367 (23):2175-2184. doi:10.1056/NEJMoa1203382

32. Gahl WA, Markello TC, Toro C, et al. The national institutes of health undiagnosed diseases program: insights into rare diseases. Gene Med. 2012;14(1):51-59. doi:10.1038/gim.0b013e318232a 005 


\section{Publish your work in this journal}

The International Journal of General Medicine is an international, peer-reviewed open-access journal that focuses on general and internal medicine, pathogenesis, epidemiology, diagnosis, monitoring and treatment protocols. The journal is characterized by the rapid reporting of reviews, original research and clinical studies

across all disease areas. The manuscript management system is completely online and includes a very quick and fair peer-review system, which is all easy to use. Visit http://www.dovepress.com/ testimonials.php to read real quotes from published authors. 